\title{
AIDA: Asteroid Impact and Deflection Assessment
}

\author{
Patrick Michel ${ }^{1}$, A. Cheng ${ }^{2}$, A. Galvez ${ }^{3}$, C. Reed ${ }^{4}$, I. Carnelli ${ }^{5}$,

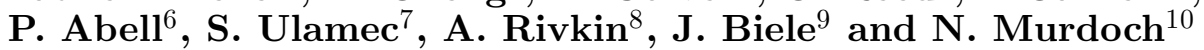 \\ ${ }^{1}$ Laboratoire Lagrange, University of Nice Sophia-Antipolis, Côte d'Azur Observatrory \\ BP 4229, 06304 Nice Cedex 4, France \\ email: michelp@oca.eu \\ ${ }^{2} \mathrm{APL} / \mathrm{JHU}$ \\ 11101 John Hopkins Road Laurel, MD 20723-6099, USA \\ email: andrew.cheng@jhuapl.edu \\ ${ }^{3}$ European Space Agency \\ 8-10 rue Mario Nikis, 75738 Paris Cedex 15, France \\ email: Andres.Galvez@esa.int \\ ${ }^{4} \mathrm{APL} / \mathrm{JHU}$ \\ 11101 John Hopkins Road Laurel, MD 20723-6099, USA \\ email: Cheryl.Reed@jhuapl.edu \\ ${ }^{5}$ European Space Agency \\ 8-10 rue Mario Nikis, 75738 Paris Cedex 15, France \\ email: Ian.Carnelli@esa.int \\ ${ }^{6}$ NASA JSC \\ Mail Code KR, 2101 NASA Parkway Houston, TX 77058-3696, USA \\ email: paul.a.abell@nasa.gov \\ ${ }^{7} \mathrm{DLR}, \mathrm{RB}-\mathrm{MC}$ \\ Linder Hhe 1, 51147 Cologne, Germany \\ email: Stephan.Ulamec@dlr.de \\ ${ }^{8} \mathrm{APL} / \mathrm{JHU}$ \\ 11101 John Hopkins Road Laurel, MD 20723-6099, USA \\ email: Andy.Rivkin@jhuapl.edu \\ ${ }^{9}$ DLR, RB-MUSC \\ Linder Hhe 29, 51147 Kln, Germany \\ email: jens.biele@dlr.de \\ ${ }^{10}$ Institut Suprieur de l'Aronautique et de l'Espace (ISAE) \\ 10 avenue Edouard Belin, BP 54032, 31055 Toulouse cedex 4, France \\ email: naomi.murdoch@isae.fr
}

\begin{abstract}
AIDA (Asteroid Impact and Deflection Assessment) is a project of a joint mission demonstration of asteroid deflection and characterisation of the kinetic impact effects. It involves the Johns Hopkins Applied Physics Laboratory (with support from members of NASA centers including Goddard Space Flight Center, Johnson Space Center, and the Jet Propulsion Laboratory), and the European Space Agency (with support from members of the french CNRS/Cte dAzur Observatory and the german DLR). This assessment will be done using a binary asteroid target. AIDA consists of two independent but mutually supporting mission concepts, one of which is the asteroid kinetic impactor and the other is the characterisation spacecraft. The objective and status of the project will be presented.
\end{abstract}

Keywords. celestial mechanics, space vehicles: instruments, minor planets, asteroids, etc. 